\title{
Engineering of Capillary-Like Structures in Tissue Constructs by
}

\section{Electrochemical Detachment of Cells}

\author{
Yuki Seto, Rina Inaba, Tomoaki Okuyama, Fumihiro Sassa, Hiroaki Suzuki, and Junji \\ Fukuda*
}

Graduate School of Pure and Applied Sciences, University of Tsukuba,

1-1-1 Tennodai, Tsukuba, Ibaraki 305-8573, Japan

*Corresponding Author: Tel.: +81-29-853-4995; Fax: +81-29-853-4490; E-mail:

fukuda@ims.tsukuba.ac.jp 
1

2

3

4

5

7

8

9

10

11

12

13

14

15

16

17

18

19

20

21

22

23

24

25

26

27

28

29

30

31

32

33

34

35

36

37

38

39

40

41

42

43

44

45

46

47

48

49

50

51

52

53

54

55

56

57

58

59

60

61

62

63

64

65

\title{
Keywords
}

Tissue engineering; blood vessel; peptide; electrochemistry; gold-thiolate bond;

\author{
collagen
}




\section{Introduction}

One of the major obstacles in engineering more complex and thick tissue

constructs such as the liver, kidney, and lung is the need to fabricate vascular networks capable of delivering oxygen and nutrients throughout the tissues $[1,2]$. Cells that are located more than a few hundred micrometers away from the nearest capillaries suffer from hypoxia and apoptosis $[3,4]$. Thus, technologies for the fabrication of spatially controlled capillaries would be required to make significant progress in tissue engineering. Most approaches to engineering vascularized tissues have relied on neovascularization from the host after transplantation. Previous attempts have involved the use of growth factor-conjugated scaffolds and extracellular matrices and genetically modified cells as vascularization factors [5, 6]. In addition, recent approaches have demonstrated that in vitro co-cultures with endothelial cells lead to formation of capillary vessel networks in engineered tissues, and that such prevascularization improves the subsequent in vivo performance of the tissue constructs [7]. Although these studies have provided evidence that a part of the vessel network is anastomosed with the host vasculature, the anastomosis processes are generally considered to be too slow to maintain cellular survival: necrotic cell death occurs within minutes or hours, whereas the invasion and anastomosis of host vessels to prevascularized vessels requires 
days or months $[8,9]$. In addition, drawbacks of the previous approaches are

inhomogeneous distribution and inadequate vascular network connections in engineered tissues, and insufficient blood flow for the supply of oxygen and nutrients throughout larger tissue constructs.

In this study, we propose a technology for the fabrication of the vascular network with a resolution of a few micrometers (Fig. 1). The key to this approach is the combination of electrochemical cell detachment and microscale technologies for uniform localization of endothelial cells on the inner surface of the capillaries. We previously reported that the cells attached to the gold surface via self-assembled alkanethiol monolayers were readily detached by applying a negative electrical potential [10]. In this study, as an alternative to alkanethiol, an oligopeptide was designed on the basis of the sequence of a natural extracellular matrix (Fig. 1A). By applying this cell detachment approach to thin gold rods aligned in a spatially controlled manner in a perfusion culture device, endothelial cells were transferred onto the surface of capillary structures (Fig. 1B). Although the structure is initially rough and primitive, angiogenesis from the surface with the endothelial cells eventually induces connections between the neighboring capillary structures in perfusion culture. This simple technique could potentially provide a fundamental tool for engineering surgically transplantable 
vascularized tissues and organs.

\section{Materials and Methods}

\subsection{Materials and reagents}

The materials used for the fabrication of culture substrates were as follows: glass wafers (\#7740; diameter, 3 in.; thickness, $500 \mu \mathrm{m}$ ) from Corning, USA; glass rods (diameter, $600 \mu \mathrm{m}$; length, $3.2 \mathrm{~cm}$ ) from Hirschmann Laborgeräte, Germany; L-cysteine from Wako Pure Chemicals Industries, Japan; and synthetic oligopeptide,

CCRRGDWLC, from Sigma-Aldrich, Japan. The reagents used for cell culture were obtained from the following commercial sources: HUVEC (CC-2517A), endothelial basal medium-2 (EBM-2, CC-3156), and SingleQuots growth supplement (CC-3162) from Cambrex Bio Science, USA; collagen type I (Cellmatrix Type I-A) from Nitta Gelatin, Inc., Japan; Ham's F12 medium from Invitrogen, Carlsbad, CA, USA; fluorescent diacetate (FDA) and ethidium bromide (EB) from Wako Pure Chemicals Industries, Osaka, Japan; phosphate buffer saline (PBS) solution from Invitrogen; phorbol 12-myristate 13-acetate (PMA) from Sigma-Aldrich. All other chemicals were purchased from Wako Pure Chemicals Industries, unless otherwise indicated.

\subsection{Design of the oligopeptide and modification on gold surface}

The oligopeptide CCRRGDWLC consists of an RGD domain in the center and 
cysteine residues at both ends. RGD interacts with cell-surface integrins expressed on

various cell types, including vascular endothelial cells $[11,12]$. Cysteine has a thiol group that chemically adsorbs onto a gold surface via a gold-thiolate bond (Fig. 1A).

Thus, the oligopeptide is designed such that it can be used for the adhesion of cells onto a gold surface. The modification process is very simple and does not require any organic chemistry. A gold surface was prepared by a sputter coating of a 1-nm layer of $\mathrm{Cr}$ and a 40-nm layer of $\mathrm{Au}$ on a glass wafer. Then the wafer was cut into small pieces of $15 \times 10$ $\mathrm{mm}$. The gold substrate was modified by immersing into 1-mM aqueous solutions of the oligopeptide for $12 \mathrm{~h}$ at room temperature. The substrate was then rinsed with pure water and sterilized with $70 \%$ ethanol for cell culture. The amount of the chemically adsorbed oligopeptide was estimated using a quartz crystal microbalance (QCM, AFFINIX-QN; Initium, Tokyo, Japan) by determining the change in frequency before and after the adsorption.

\subsection{Cyclic voltammetry}

Cyclic voltammetry was employed to determine the reductive potential for desorption of the oligopeptide adsorbed on the gold surface. Immediately before cyclic voltammetry testing, an electrolyte solution containing $0.5 \mathrm{M} \mathrm{KOH}$ was deoxygenated by bubbling nitrogen gas for $20 \mathrm{~min}$. The oligopeptide-modified gold substrate, a 
$\mathrm{Ag} / \mathrm{AgCl}$ reference electrode (\#2080 A; Horiba, Tokyo, Japan), and a platinum auxiliary

electrode were set in the electrolyte solution and connected to an electrochemical

measurement system (AUTOLAB; Metrohm Autolab, The Netherlands). In this study,

all potential values refer to those measured with respect to a $\mathrm{Ag} / \mathrm{AgCl}$ electrode. $\mathrm{A}$

cyclic voltammogram was recorded at the scanning rate of $20 \mathrm{mV} / \mathrm{s}$ from 0 to $-1.0 \mathrm{~V}$.

\subsection{Cell preparation}

HUVECs were cultured in EBM-2 supplemented with SingleQuots growth

supplement (vascular endothelial growth factor, fibroblast growth factor-B, and fetal

bovine serum) at $37{ }^{\circ} \mathrm{C}$ and $5 \% \mathrm{CO}_{2}$ in a humidified incubator. The medium was

changed every other day. Each passage was conducted with a solution of $0.25 \%$ trypsin

and $0.02 \%$ ethylenediamine tetraacetic acid (EDTA) after 3 to 4 days, when the cells

were resuspended in fresh medium and diluted 1:3. Cells from passages 3 through 8

were used for experiments.

\subsection{Detachment of cells along with electrochemical desorption of the oligopeptide}

The substrate modified with the oligopeptide was placed in a typical 35-mm dish, and HUVECs were seeded at a density of $1.5 \times 10^{5}$ cells in 2 -mL culture medium. The cells were cultured for $18 \mathrm{~h}$ at $37{ }^{\circ} \mathrm{C}$ and $5 \% \mathrm{CO}_{2}$ in a humidified incubator. Then the substrate was washed with PBS and connected to a potentiostat (HA-151; 
Hokuto-Denko, Japan). After applying a potential of $-1.0 \mathrm{~V}$ for 1, 2, 3, 5, and $7 \mathrm{~min}$, the substrate was washed gently and the remaining cells were counted under a phase contrast microscope (IX-71; Olympus Co., Japan). For comparison, the same experiments were conducted without the oligopeptide (cells attached directly to a gold surface) or by applying a potential of $-0.5 \mathrm{~V}$, which is smaller than that required for desorption of the oligopeptide.

To transfer cells to the collagen gel using this approach, adherent cells on the substrate were covered with collagen solution. The collagen solution was previously prepared by mixing type I collagen $(3.0 \mathrm{mg} / \mathrm{mL}), 10$-fold concentrated Ham's F12 medium, and reconstitution buffer containing 0.05 N NaOH, $200 \mathrm{mM}$ HEPES, and $2.2 \% \mathrm{NaHCO}_{3}$ at a ratio of 8:1:1 on ice. After gelation of the collagen, the gel layer was peeled off from the substrate after applying a potential of $-1.0 \mathrm{~V}$ for $5 \mathrm{~min}$. Cell viability after the detachment was evaluated with a live/dead fluorometric assay with FDA and EB [13].

\subsection{Fabrication of capillary-like structures}

A thin gold rod was prepared by a sputter coating of layers of $\mathrm{Cr}$ and $\mathrm{Au}$ on a glass stick of $600-\mu \mathrm{m}$ diameter. Similar to the glass wafer surface, the surface of the gold rod was modified with the oligopeptide. The modified gold rod was then rinsed with pure 
water and sterilized with $70 \%$ ethanol. The gold rods were placed in a cell-nonadherent 35-mm dish (Techno Plastic Products, Switzerland) and HUVECs were seeded at a density of $3.0 \times 10^{5}$ cells in $2-\mathrm{mL}$ culture medium. The cells were attached to the gold rods and grown to reach confluence for 3 to 4 days.

The chamber for perfusion culture (Fig. 1B) was fabricated with a poly(methyl methacrylate) plate using computer-aided laser machining (Laser PRO C180; GCC, Taiwan). The volume of the chamber was $1.5 \mathrm{~mL}$. The chamber had three pairs of holes of $800 \mu \mathrm{m}$ in diameter at intervals of $500 \mu \mathrm{m}$ for the guidance of the gold rods. The gold rods with cells were fixed in the chamber, and $1.5 \mathrm{~mL}$ of the collagen solution was then poured into the chamber. After the gelation of the collagen, the rods were carefully extracted by applying a potential of $-1.0 \mathrm{~V}$ for $5 \mathrm{~min}$. Then the chamber was connected to a microsyringe pump and culture medium was perfused at $10 \mu \mathrm{L} / \mathrm{min}$. To accelerate spontaneous vascularization, PMA was added to the culture medium to give a final concentration of $20 \mathrm{ng} / \mathrm{mL}[14,15]$.

\subsection{Scanning electron microscopy}

To observe cells on the gold rods under a scanning electron microscope (SEM), the culture was washed with PBS thrice and fixed with a mixed solution of $2.5 \%$ glutaraldehyde and 2\% formaldehyde in PBS for $1 \mathrm{~h}$ at room temperature. Then, the 
culture was washed with PBS and fixed with $1 \%$ osmium tetroxide in PBS for $1 \mathrm{~h}$ at

$4{ }^{\circ} \mathrm{C}$. The culture was washed with purified water and dehydrated with a graded ethanol series from $30 \%$ to $90 \%$ on ice and absolute ethanol substitution three times at room temperature. The solution was further substituted with $100 \% t$-butanol, which was frozen at $4{ }^{\circ} \mathrm{C}$ and dried by vacuum freeze-drying. The cells were observed under a SEM (ED-SEM; JEOL, Japan) operated at $5 \mathrm{kV}$.

\section{Results and Discussion}

\subsection{Electrical potential required for desorption of the oligopeptide}

The amount of oligopeptide adsorbed on the gold surface was estimated to be $8.7 \pm$ $1.4 \mathrm{ng} / \mathrm{cm}^{2}$ from three independent measurements with a QCM. This value corresponds to $159 \mathrm{pmol} / \mathrm{cm}^{2}$, which is high enough for the adhesion of cells (typical cases have required a maximum of $\sim 20 \mathrm{pmol} / \mathrm{cm}^{2}$ ) [16]. Cyclic voltammetry showed that the peak potentials for reductive desorption of the oligopeptide and cysteine were approximately $-0.86 \mathrm{~V}$ and $-0.70 \mathrm{~V}$, respectively (Fig. 2). The relationship between the molecular configurations and the peak potentials required for cleaving the gold-thiolate bonds has been previously discussed in detail in experiments involving self-assembled alkanethiol monolayers $[17,18]$. The shape and potential of the peak are considered to be closely associated with a van der Waals interaction between the adsorbed molecules and the 
steric structure of the molecules $[19,20]$. As seen in Fig. 2, the peak for the oligopeptide is wider than that for cysteine, suggesting that the oligopeptide formed a relatively loose layer on the surface and had weak interactions with neighboring oligopeptides, probably because of its bulky structure. In general, the number of adsorbed molecules can be estimated from both the peak area in a cyclic voltammogram and by using a QCM. The amount of oligopeptide estimated by using a QCM was five times greater than that determined from the cyclic voltammogram. This fact suggests that the oligopeptide possibly forms intermolecular cross-links via disulfide binding, and a part of the thiol group bonds to the gold surface. Although the details of the surface chemistry are still unclear, on the basis of the results that the oligopeptide desorbed in the potential range of -0.8 to $-1.0 \mathrm{~V},-1.0 \mathrm{~V}$ was used for detachment of cells in the subsequent experiments.

\subsection{Change in HUVEC morphology during cell detachment}

HUVECs were readily attached via the oligopeptide and spreaded out on the gold surface (Fig. 3A). There was no significant difference in the number of cells attached onto the gold surface with or without modification with the oligopeptide (1.0 to $1.5 \times$ $10^{4}$ cells $/ \mathrm{cm}^{2}$ ). At $18 \mathrm{~h}$ of culture, the cells attached onto the gold surface with the oligopeptide were detached by applying a potential of $-1.0 \mathrm{~V}$. Figures $3 \mathrm{~B}-\mathrm{J}$ show the 
change in HUVEC morphology at 20-s intervals during the detachment. The cells were gradually detached from the adhesive ends and appeared bright and round after the application of the potential. These cells were identified to be detached after gentle pipetting (Fig. 3K).

To quantitatively analyze the detachment, the number of cells that remained on the surface was counted at 1, 3, 5, and 7 min after the application of the potential (Fig. 4). When the oligopeptide and an electrical potential of $-1.0 \mathrm{~V}$ were used, more than $90 \%$ of the cells detached from the surface within $7 \mathrm{~min}$. On the other hand, the behavior of the cells was clearly different in the control experiments (conditions of no oligopeptide or application of a potential of $-0.5 \mathrm{~V}$, which is smaller than that required to cleave the gold-thiolate bond). These results suggest that cell detachment occurs mainly due to the electrically dependent desorption of the oligopeptide.

There are a few reports regarding the electrochemical detachment of cells. A self-assembled monolayer with electroactive sites that respond to electrical potentials has been used for micropatterning of cells by detachment of cells from a selective region [21]. We have also previously shown that cells or cell sheets on the gold surface modified with alkanethiol could be detached by application of a potential [10]. To promote the adhesion of cells, the carboxyl terminal of alkanethiol has been coupled 
with an RGD peptide through carbodiimide-mediated cross-linking. Multilayered polyelectrolyte films formed by layer-by-layer deposition have also been used to detach cell sheets [22]. In this approach, the application of the relatively large potential, $-1.8 \mathrm{~V}$, induces electrolysis of water and a local $\mathrm{pH}$ change, leading to the dissolution of the polyelectrolyte film and the detachment of cell sheets. An unsolved issue in these previous approaches is that molecules and other chemicals may be contained in the detached tissues and potentially could cause inflammatory responses in the body after transplantation. To alleviate this problem, we used an oligopeptide in this study. Since the oligopeptide contains an RGD sequence and spontaneously bonds to a gold surface, the culture surface could be prepared without the use of coupling agents, simply by immersing a gold-coated substrate in a peptide solution. This may be advantageous for the widespread use of this technology in biological and medical laboratories.

\subsection{Transfer of cells from the gold surface to collagen gel}

After the collagen solution was poured and gelated on HUVECs on the gold surface with or without modification with the oligopeptide, a potential of $-1.0 \mathrm{~V}$ was applied for $5 \mathrm{~min}$ and the gel layer was peeled off. The cells that were attached to the gold surface with the oligopeptide were transferred to the gel, whereas in the case where the oligopeptide was not used only a few cells were transferred. Figures 5B and C show the 
surface of the substrates after peeling off the gel when the oligopeptide was and was not used, respectively. We then evaluated the viability of the transferred cells in the gel by staining live cells with FDA (green) and dead cells with EB (red). Although the gelation may have trapped the cells in proximity to the electrode surface during the potential application, almost all the cells transferred were viable and few dead cells were observed in the gel (Fig. 5D). In the absence of the oligopeptide, only a small number of cells were transferred to the gel, some of which were dead cells (Fig. 5E). Figure 5F shows the cells cultured for $24 \mathrm{~h}$ after the transfer into the gel. The cells grew and formed connections with each other on the gel. These results show that this approach (using an electrochemical reaction) can be used to transfer cells to the collagen gel noninvasively.

\subsection{Fabrication of capillary-like structures in collagen gel}

We applied this approach for the fabrication of capillary-like structures by transferring cells from thin gold rods to the inner surface of capillaries in a collagen gel (Fig. 1B). The rods covered with HUVECs (Figs. 6A and B) were aligned in the chamber and a collagen solution was poured and allowed to form a gel. After applying a potential of $-1.0 \mathrm{~V}$ for $5 \mathrm{~min}$, the rods were carefully extracted from the chamber through guide holes, resulting in the formation of capillaries enveloped with HUVECs. 
The capillaries were formed at $\sim 500-\mu \mathrm{m}$ intervals (Fig. $6 \mathrm{C}$ ) and were $16 \mathrm{~mm}$ in length (Fig. 6D).

The guide holes were then connected to silicone tubes and the culture medium containing PMA was perfused through the capillaries at a flow rate of $10 \mu \mathrm{L} / \mathrm{min}$. Although the perfusion of culture medium was not necessarily required for oxygen supply in this experiment, when parenchymal cells such as hepatocytes are seeded in the collagen, a prompt initiation of culture medium flow will be required to satisfy their oxygen demand. Another approach was reported wherein a channel structure was constructed in a collagen gel and endothelial cells were then seeded into it to form a capillary-like structure. However, that approach required that the flow of culture medium was stopped for a while and the device was rotated to let cells attach to the inner surface of the channel, thereby making it difficult to stably supply oxygen to parenchymal cells [23].

The flow rate used in this study was relatively low in comparison with those observed in in vivo peripheral vessels. The shear stress caused by the flow was calculated to be $0.12 \mathrm{dyn} / \mathrm{cm}^{2}$ in this study, whereas it was $1-5 \mathrm{dyn} / \mathrm{cm}^{2}$ in the venules [24]. During the perfusion culture, the HUVECs were oriented in the direction of the stream at $6 \mathrm{~h}(\mathrm{Fig}$. $6 \mathrm{~F})$ and partly began to migrate into the collagen gel at $48 \mathrm{~h}$ at the 
earliest (Fig. 6G). The formation of luminal structures in the collagen gel was dependent

on the time and position. In a representative case, the HUVECs began to sprout at 4 days of perfusion culture, and reached the neighboring channels and bridged them with a luminal structure at $\sim 7$ days (Fig. 6J).

PMA was required for the induction of sprouting and formation of luminal structures. Although the culture medium contained vascular endothelial growth factor and fibroblast growth factor-B, capillary formation was not observed in a highly reproducible manner, which was in contrast to that observed in the case of the medium supplemented with PMA. PMA is a potent promoter of tumor development and progression and activates the signal transduction enzyme protein kinase $\mathrm{C}$ because of its structural similarity to one of the natural activators, diacylglycerol [25, 26]. Although PMA is currently employed in phase-I clinical trials for the treatment of patients with hematologic cancer or bone marrow disorder [27], its use should be avoided to prevent the development of its clinical side effects. Because HUVECs are known to form capillaries in a collagen gel when cultured in medium containing the abovementioned growth factors, optimization of the concentrations of these growth factors or their embedment in the collagen gel may lead to the development of a more suitable approach, which will eliminate the need for PMA. 


\section{Conclusion}

This study demonstrated an electrochemical technique to fabricate capillaries whose internal surface was covered with vascular endothelial cells in collagen gel. In this approach, HUVECs were attached to a gold surface via an oligopeptide. The cells were detached by applying a negative potential that reductively cleaved the gold-thiolate bonds. This technique allowed for detachment of more than $90 \%$ of the attached cells within 7 min of applying the negative potential, and could be used to detach cells not only from flat surfaces but also from thin rods. To fabricate the capillaries, a gold rod enveloped with HUVECs was inserted into a collagen gel. The rod was then extracted by applying a potential of $-1.0 \mathrm{~V}$, resulting in the formation of a capillary, which was enveloped with endothelial cells, in the collagen gel. During the subsequent perfusion culture, luminal structures were formed from the HUVECs lining the capillary and they bridged the neighboring capillaries to each other. This approach has potential for engineering vascularized tissues capable of delivering oxygen and nutrients to the entire tissue construct.

\section{Acknowledgments}

This research has been supported by MEXT (Grant-in-Aid for Young Scientists (A), 20686056), Ministry of Health, Labor and Welfare (H20-Saisei-wakate-010), and 
1

2

3

4

5

6

7

8

9

10

11

12

13

14

15

16

17

18

19

20

21

22

23

24

25

26

27

28

29

30

31

32

33

34

35

36

37

38

39

40

41

42

43

44

45

46

47

48

49

50

51

52

53

54

55

56

57

58

59

60

61

62

63

64

65

NEDO (Industrial Technology Research Grant Program, 06A06014a). 


\section{References}

1. Moon JJ, West JL. Vascularization of engineered tissues: Approaches to promote angiogenesis in biomaterials. Curr Top Med Chem 2008;8(4):300-310.

2. Khademhosseini A, Langer R. Microengineered hydrogels for tissue engineering. Biomaterials 2007;28(34):5087-5092.

3. Patel ZS, Mikos AG. Angiogenesis with biomaterial-based drug- and cell-delivery systems. J Biomater Sci Polym Ed 2004;15(6):701-726.

4. Fukuda J, Mizumoto H, Nakazawa K, Kajiwara T, Funatsu K. Hepatocyte organoid culture in elliptic hollow fibers to develop a hybrid artificial liver. Int J Artif Organs 2004;27(12):1091-1099.

5. Richardson TP, Peters MC, Ennett AB, Mooney DJ. Polymeric system for dual growth factor delivery. Nat Biotechnol 2001;19(11):1029-1034.

6. Lu YX, Shansky J, Del Tatto M, Ferland P, Wang XY, Vandenburgh H. Recombinant vascular endothelial growth factor secreted from tissue-engineered bioartificial muscles promotes localized angiogenesis. Circulation 2001;104(5):594-599.

7. Levenberg S, Rouwkema J, Macdonald M, Garfein ES, Kohane DS, Darland DC, et al. Engineering vascularized skeletal muscle tissue. Nat Biotechnol 2005;23(7):879-884.

8. Croll TI, Gentz S, Mueller K, Davidson M, O’Connor AJ, Stevens GW, et al. Modelling oxygen diffusion and cell growth in a porous, vascularising scaffold for soft tissue engineering applications. Chem Eng Sci 2005;60(17):4924-4934.

9. Chen X, Aledia AS, Ghajar CM, Griffith CK, Putnam AJ, Hughes CC, et al.

Prevascularization of a fibrin-based tissue construct accelerates the formation of functional anastomosis with host vasculature. Tissue Eng Part A 2009;15(6):1363-1371.

10. Inaba R, Khademhosseini A, Suzuki H, Fukuda J. Electrochemical desorption of self-assembled monolayers for engineering cellular tissues. Biomaterials 2009;30(21):3573-3579.

11. Healy JM, Haruki M, Kikuchi M. Preferred motif for integrin binding identified using a library of randomized RGD peptides displayed on phage. Protein Pept Lett 1996;3(1):23-30.

12. McMillan R, Meeks B, Bensebaa F, Deslandes Y, Sheardown H. Cell adhesion peptide modification of gold-coated polyurethanes for vascular endothelial cell adhesion. J Biomed Mater Res 2001;54(2):272-283.

13. Fukuda J, Sakiyama R, Nakazawa K, Ijima H, Yamashita Y, Shimada M, et al. Mass 
preparation of primary porcine hepatocytes and the design of a hybrid artificial liver module using spheroid culture for a clinical trial. Int J Artif Organs 2001;24(11):799-806.

14. Gamble JR, Matthias LJ, Meyer G, Kaur P, Russ G, Faull R, et al. Regulation of in vitro capillary tube formation by anti-integrin antibodies. J Cell Biol 1993;121(4):931-943.

15. Montesano R, Orci L. Tumor-promoting phorbol esters induce angiogenesis in vitro. Cell 1985;42(2):469-477.

16. Houseman BT, Mrksich M. The microenvironment of immobilized Arg-Gly-Asp peptides is an important determinant of cell adhesion. Biomaterials 2001;22(9):943-955.

17. Walczak MM, Popenoe DD, Deinhammer RS, Lamp BD, Chung CK, Porter MD. Reductive desorption of alkanethiolate monolayers at gold-A measure of surface coverage. Langmuir 1991;7(11):2687-2693.

18. Imabayashi S, Iida M, Hobara D, Feng ZQ, Niki K, Kakiuchi T. Reductive desorption of carboxylic-acid-terminated alkanethiol monolayers from $\mathrm{Au}(111)$ surfaces. J Electroanal Chem 1997;428(1-2):33-38.

19. Widrig CA, Chinkap C, Porter MD. The electrochemical desorption of n-alkanethiol monolayers from polycrystalline Au and Ag electrodes. J Electroanal Chem 1991;310(1-2):335-359.

20. Imabayashi S, Hobara D, Kakiuchi T, Knoll W. Selective replacement of adsorbed alkanethiols in phase-separated binary self-assembled monolayers by electrochemical partial desorption. Langmuir 1997;13(17):4502-4504.

21. Yeo WS, Mrksich M. Electroactive self-assembled monolayers that permit orthogonal control over the adhesion of cells to patterned substrates. Langmuir 2006;22(25):10816-10820.

22. Guillaume-Gentil O, Akiyama Y, Schuler M, Tang C, Textor M, Yamato M, et al. Polyelectrolyte coatings with a potential for electronic control and cell sheet engineering. Adv Mater 2008;20(3):560-565.

23. Takei T, Sakai S, Ono T, Ijima H, Kawakami K. Fabrication of endothelialized tube in collagen gel as starting point for self-developing capillary-like network to construct three-dimensional organs in vitro. Biotechnol Bioeng 2006;95(1):1-7.

24. Pries AR, Secomb TW, Gaehtgens P. Design principles of vascular beds. Circ Res 1995;77(5):1017-1023.

25. Castagna M, Takai Y, Kaibuchi K, Sano K, Kikkawa U, Nishizuka Y. Direct Activation of Calcium-Activated, Phospholipid-Dependent Protein-Kinase by 
Tumor-Promoting Phorbol Esters. J Biol Chem 1982;257(13):7847-7851.

26. Murphy RLW, Smith ME. Effects of Diacylglycerol and Phorbol Ester on

Acetylcholine-Release and Action at the Neuromuscular-Junction in Mice. Br J Pharmacol 1987;90(2):327-334.

27. http://www.clinicaltrials.gov/ct/show/NCT00004058 


\section{Figure Captions}

Figure 1. Cell detachment along with reductive desorption of the oligopeptide. (A) The oligopeptide CCRRGDWLC was chemically adsorbed onto a gold surface at both terminals via a gold-thiolate bond. Cells attached to the surface were detached during the reductive desorption of the oligopeptide. (B) Thin gold rods enveloped with cells were aligned in a spatially controlled manner in a chamber. By applying a potential and extracting the rods from the collagen gel, the cells were transferred onto the surface of capillaries. The device was connected to a microsyringe pump for perfusion of culture medium. The capillaries connect with each other to form luminal structures.

Figure 2. Cyclic voltammogram obtained during the reductive desorption of the oligopeptide. The current peaks for the oligopeptide $(\circ)$ and L-cysteine $(\bullet)$ were -0.86 $\mathrm{V}$ and $-0.71 \mathrm{~V}$, respectively. Cyclic voltammograms were recorded at a scanning rate of $20 \mathrm{mV} / \mathrm{s}$ with respect to a $\mathrm{Ag} / \mathrm{AgCl}$ reference electrode. The working electrode area was $1.0 \mathrm{~cm}^{2}$

Figure 3. Detachment of HUVECs from the gold surface. (A) Cells were readily attached to the gold surface modified with the oligopeptide during culturing for $12 \mathrm{~h}$. 
(B)-(J) The change in cell morphology during the application of the electrical potential was observed at 20-s intervals. The cells were gradually detached from the adhesive ends and appeared bright and round after the application of the potential. (K) The cells were withdrawn into a micropipette and found to be detached.

Figure 4. Change in the number of HUVECs remaining on the gold surfaces.

Approximately $90 \%$ of cells were detached within 7 min along with the desorption of the oligopeptide $(\bullet)$. Few cells detached from the surface in the absence of the oligopeptide (i.e., cells were directly attached to a gold surface [०] and a potential of $-0.5 \mathrm{~V}$, which is smaller than that required to cleave the gold-thiolate bond [ $\square]$ ). The error bars indicate SD calculated from nine independent experiments for each plot.

Figure 5. Transfer of HUVECs to a collagen gel. Cells that attached to the gold surface modified with the oligopeptide (A) were transferred to a collagen gel, and few cells remained on the surface after potential application and peeling off the gel (B). Most of the cells remained attached on the gold surface that was not modified with the peptide even after the same potential was applied and the gel was peeled off (C). The cells transferred to the gel were viable, and few cells that were attached onto the surface 
modified with the peptide died (D), whereas only few cells that were attached onto the surface without peptide modification were transferred to the gel (E). The transferred cells grew and spread out on the gel at 1 day of culture $(F)$.

Figure 6. Fabrication of capillary-like structures in collagen gel and formation of vascular networks. HUVECs were attached to the gold rod via the oligopeptide and grown to reach confluence at 3 days of culture $(\mathrm{A}, \mathrm{B})$. The capillaries were formed at $500-\mu \mathrm{m}$ intervals in a collagen gel (C). HUVECs were transferred throughout the capillaries (16-mm length) from the inlet to the outlet (D). The transferred HUVECs (E) oriented in the direction of the stream at $6 \mathrm{~h}$ of perfusion culture $(\mathrm{F})$. HUVECs began to partly migrate into the collagen gel as early as $48 \mathrm{~h}(\mathrm{G})$. For the most part, HUVECs grew to form a dense cell layer on the surface at 2 days $(\mathrm{H})$, and began to migrate into the collagen gel at $\sim 4$ days (I). At $\sim 7$ days, the luminal structures extended to the neighboring channels and bridged them $(\mathrm{J})$. 
Figure 1

Click here to download high resolution image

Fig. 1 Y. Seton, et al.

A
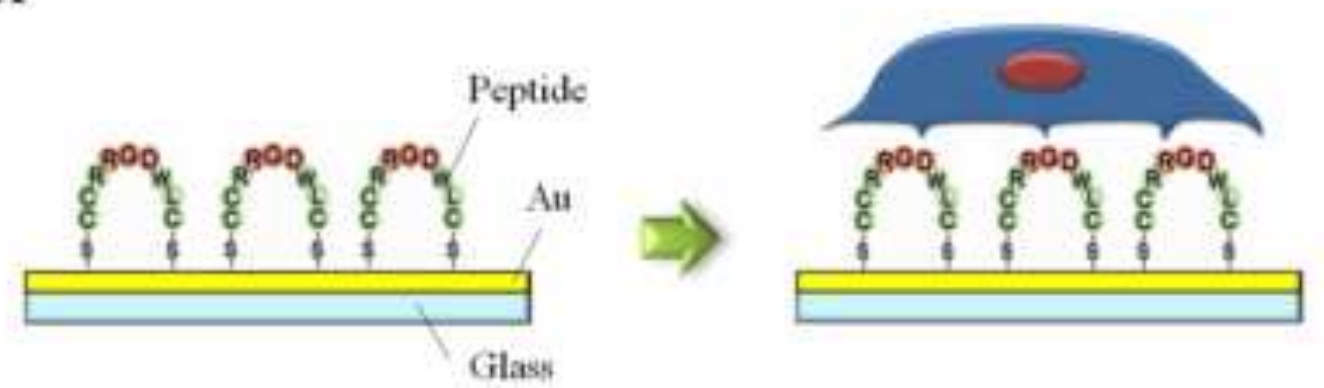

Potential application

$\Rightarrow$

B
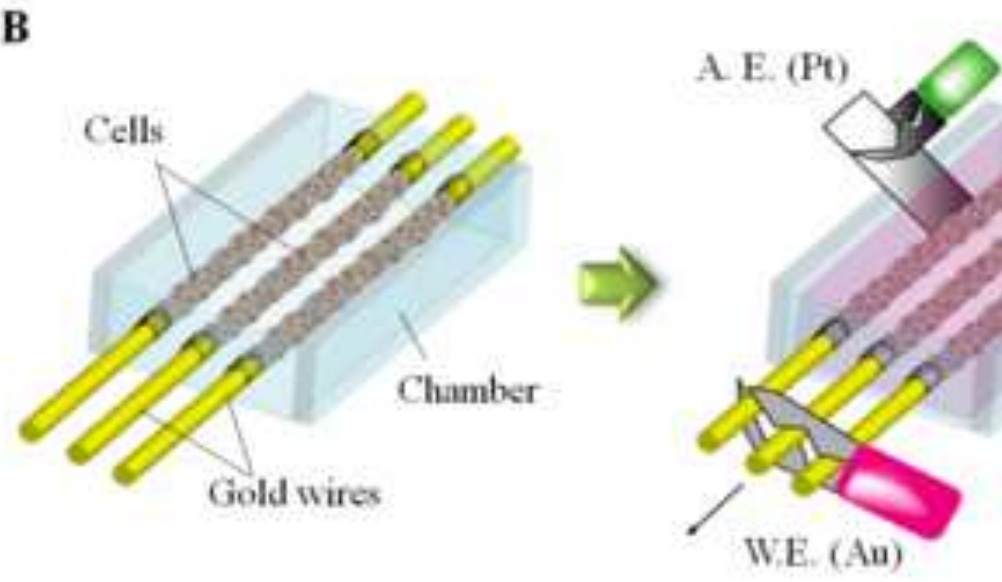

Culture medium

RE. ( $\mathrm{Ag} \mathrm{AgCl})$

Collagen gel

1
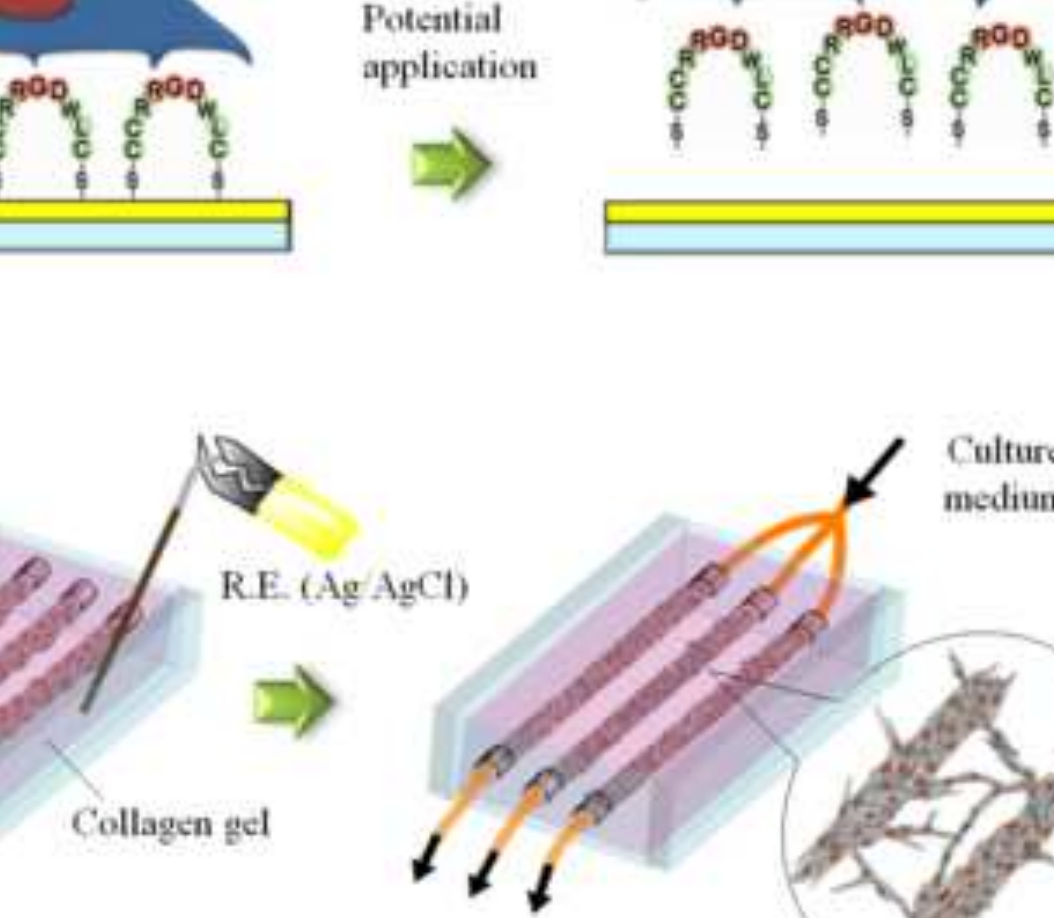

$\beta$

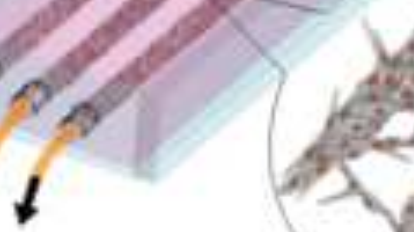

W.E. (Au) 


\section{gure 2}

Click here to download high resolution image

Fig. 2 Y. Seto, et al.

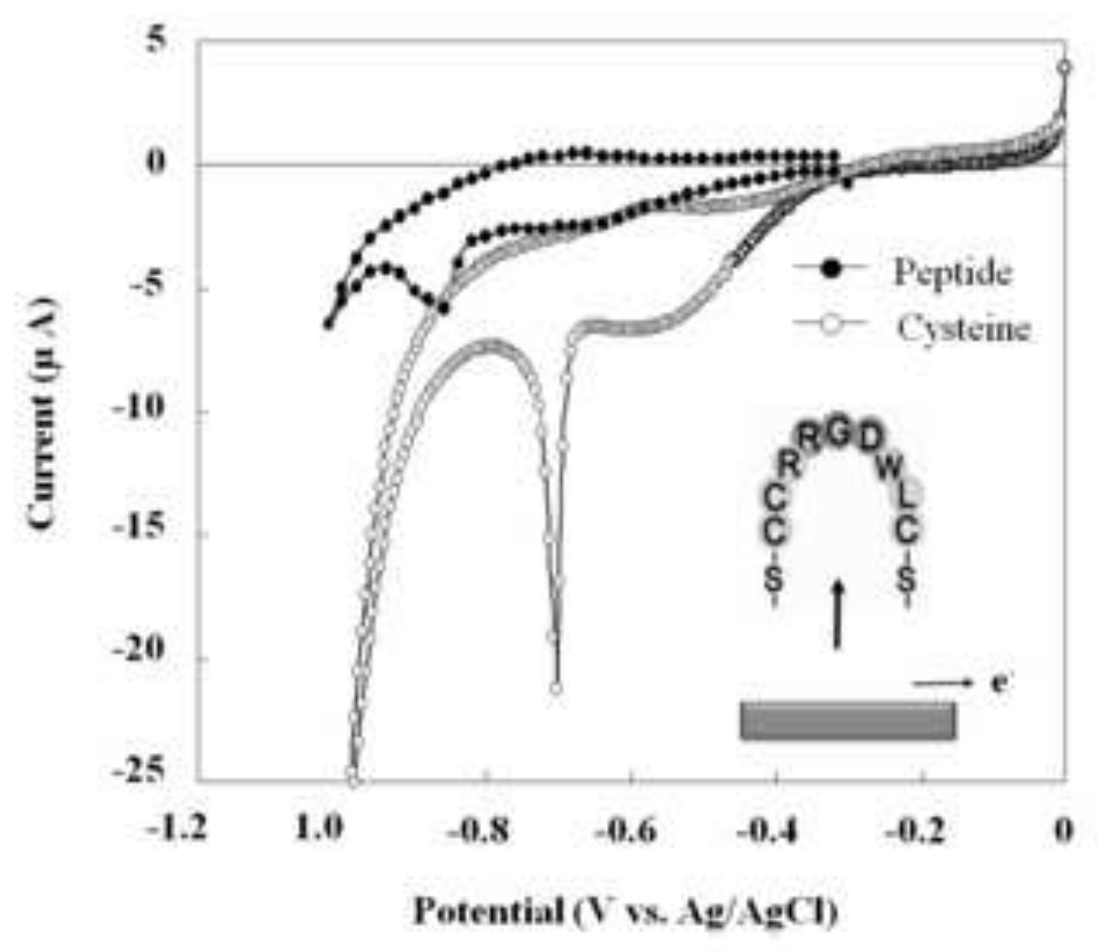


Fig. 3 Y. Seto, et al.
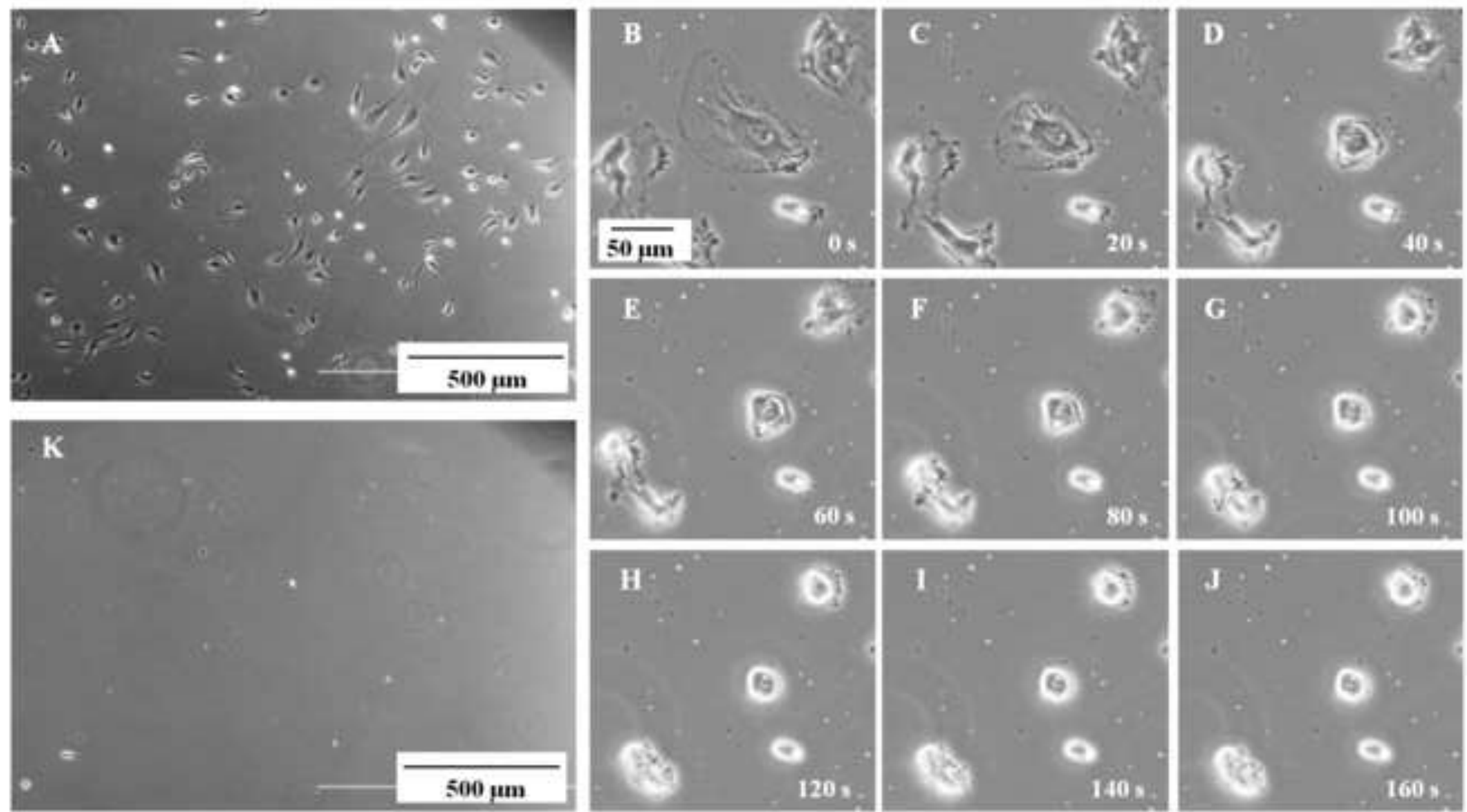

G:
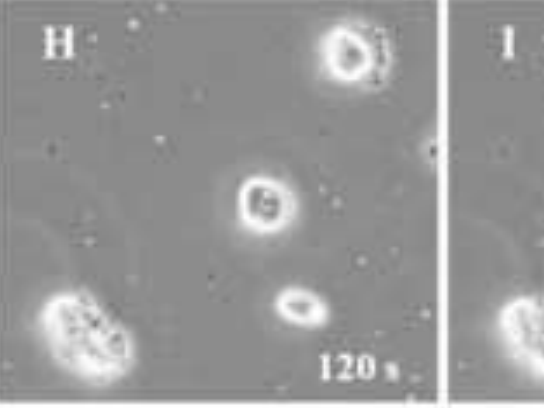

$120 \times$.

140. 
Click here to download high resolution image

Fig. 4 Y. Seto, et al.

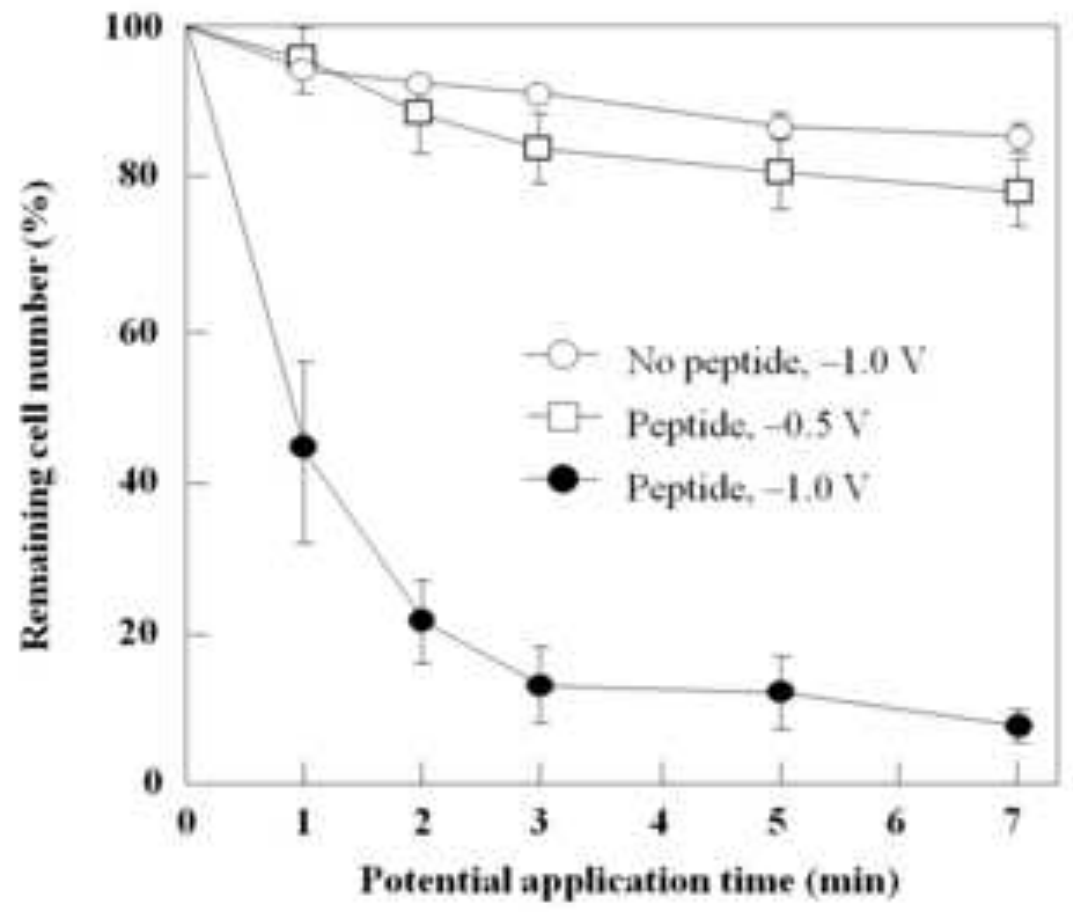


Fig. 5 Y. Seto, et al.
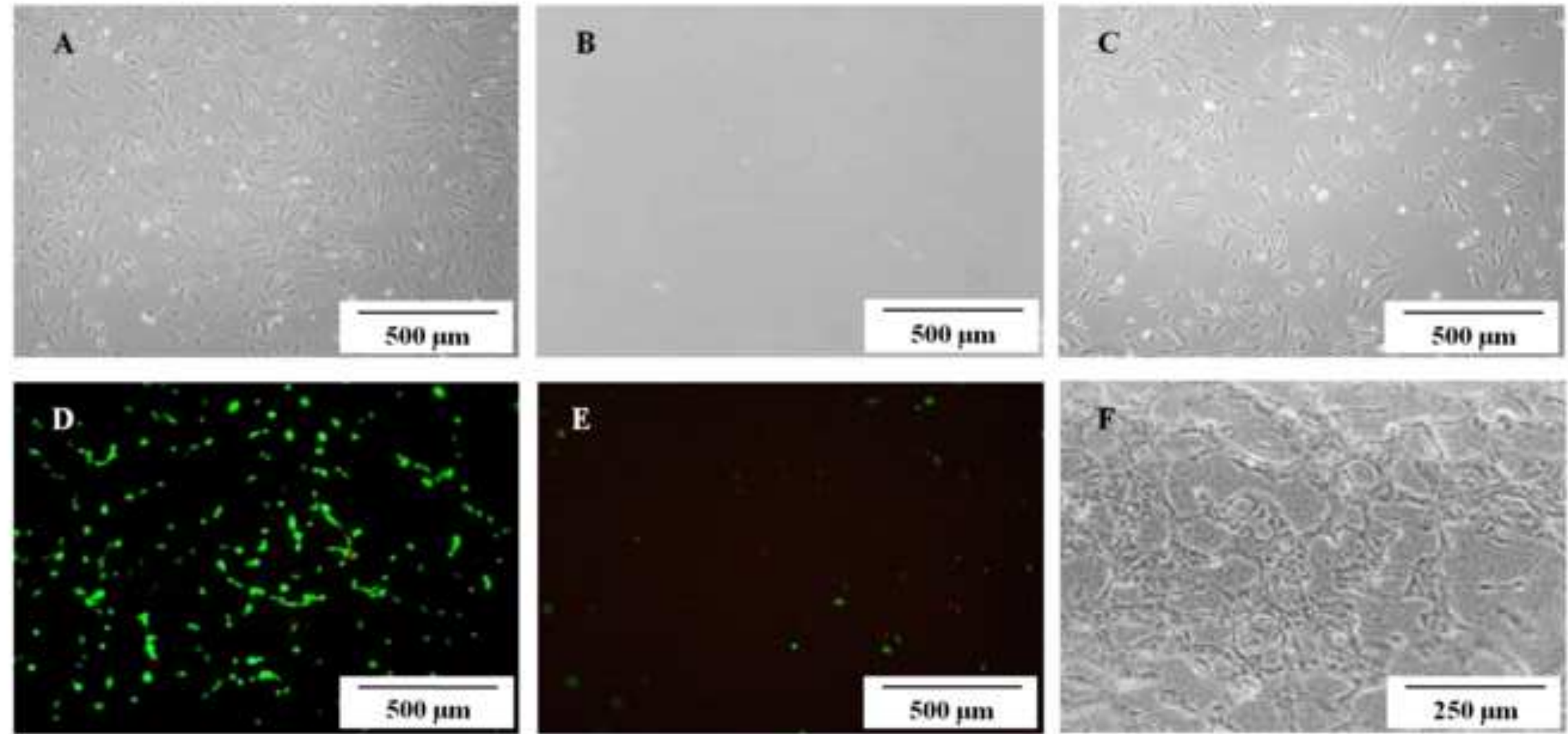
Click here to download high resolution image

Fig. 6 Y. Seto, et al.
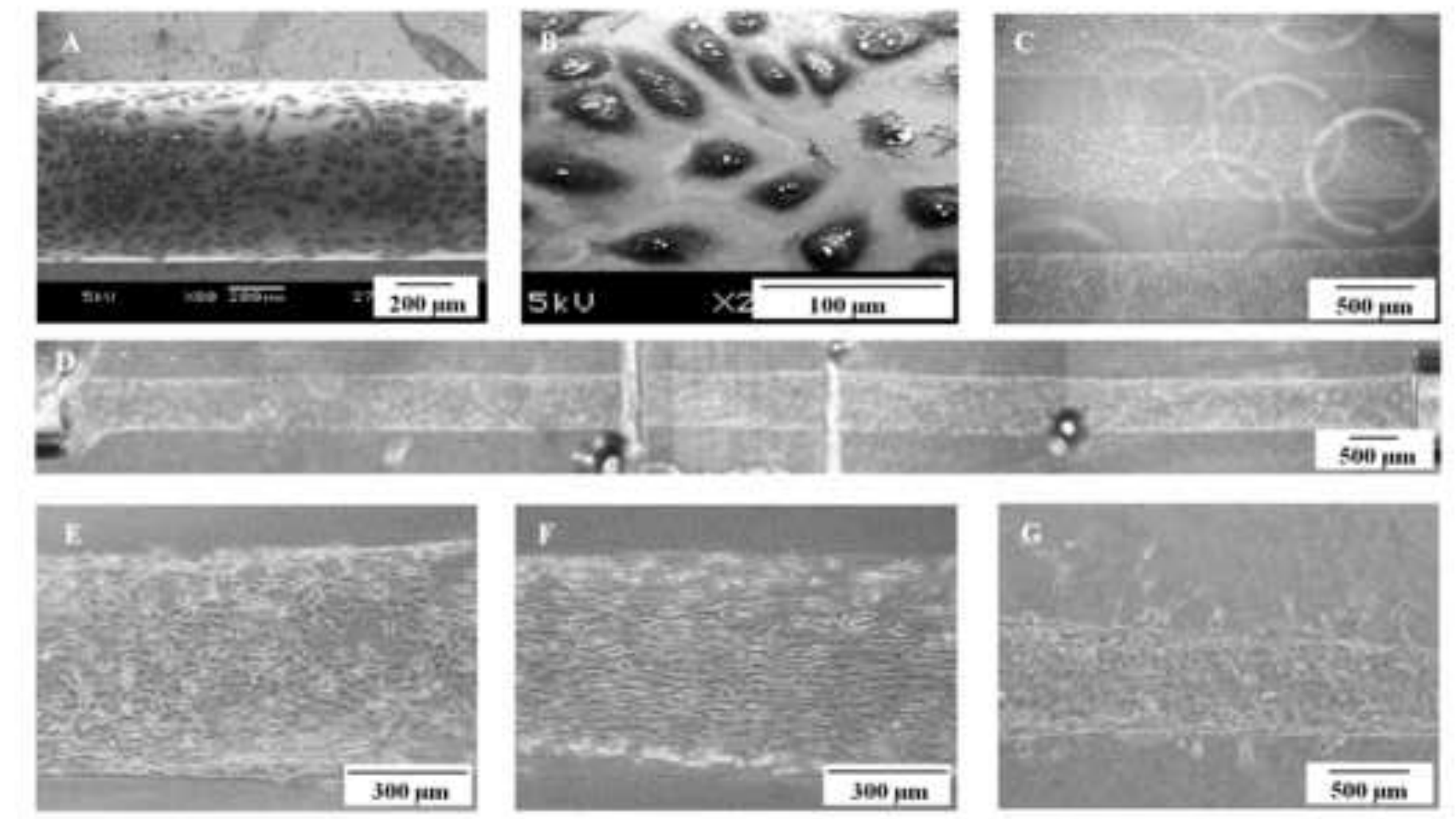

00 min
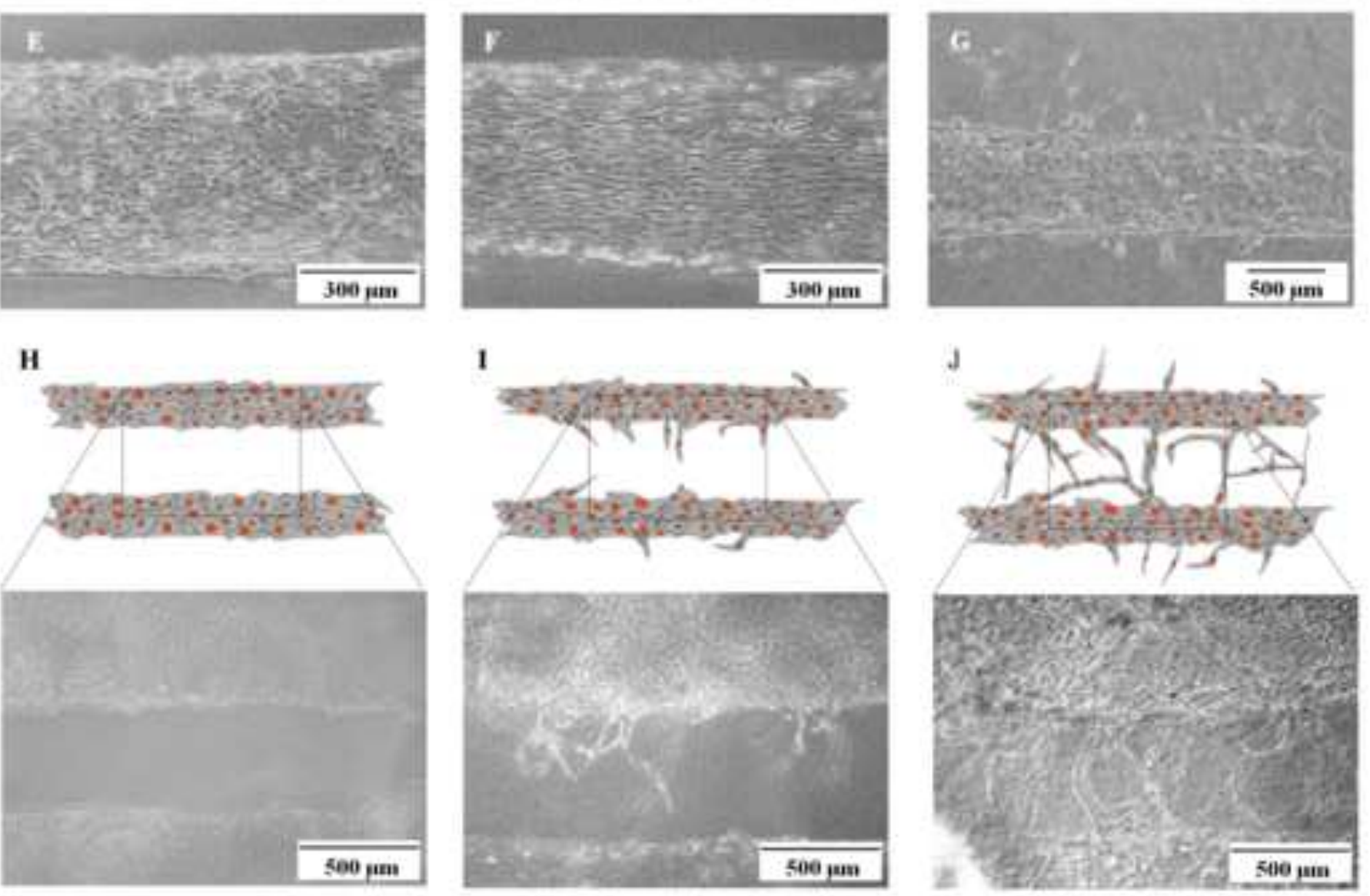
Movie/Animation
Click here to download Movie/Animation: detachment_3min.avi i

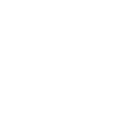
(a) (1) 